\title{
Mesh generation in curvilinear domains using high-order elements
}

\author{
S. J. Sherwin and J. Peiró \\ Department of Aeronautics, \\ Imperial College of Science, Technology \& Medicine, \\ Prince Consort Road, South Kensington, London, SW7 $2 B Y, U K$
}

\section{SUMMARY}

The ability to construct suitable computational meshes is currently a significant limiting factor in the development of compact high-order algorithms in very complex geometries. Compact high-order algorithms such as spectral element or $p$-type finite element techniques offer the potential of high accuracy if the solution is smooth and a well behaved mapping exists between the local sub-domains and a standard region. In this paper we address the problem of coupling high-order algorithms with unstructured mesh generation and CAD representation techniques. We present three complementary strategies to alleviate the problem of generation of elemental regions with singular elemental mappings. The first strategy investigates the influence of an anisometric parametric surface representation on the quality of high-order unstructured meshes. We show that a straightforward splitting of a standard linear mesh for use in a high-order method can lead to the generation of distorted, possibly invalid, meshes even for simple computational domains such as cubes. The second strategy uses hybrid meshes applying prismatic elements near curvilinear boundaries. In the third and final strategy we investigate the use of curvature driven surface discretisation to incorporate higher order information about the surface into the mesh generation. Using the computational reconstruction of the arterial bypass graft as an example computational domain we demonstrate how the combination of all three strategies leads to a valid high-order discretisation of the computational domain. Copyright (C) 2000 John Wiley \& Sons, Ltd.

KEY WORDS: high order surface representation, hybrid meshing, curvature based adaption, high order elements

The desire to solve unsteady flow problems for critical engineering problems has motivated the development of accurate algorithms suitable for geometrically complex problems. Such algorithms have been developed from different numerical techniques such as compact finite differences [1], high-order finite volume schemes and spectral/hp element methods $[2,3]$. Although these techniques offer the potential for high accuracy this is achieved at the expense of greater implementation complexity. There is therefore a need for automatic methods that hide the complexity of the implementation details. These algorithms should also be robust in the sense that non-expert users should be able to handle a wide range of geometrically complex problems without user intervention.

${ }^{*}$ Correspondence to: Department of Aeronautics, Imperial College of Science, Technology \& Medicine,Prince Consort Road, South Kensington,London, SW7 2BY,UK 


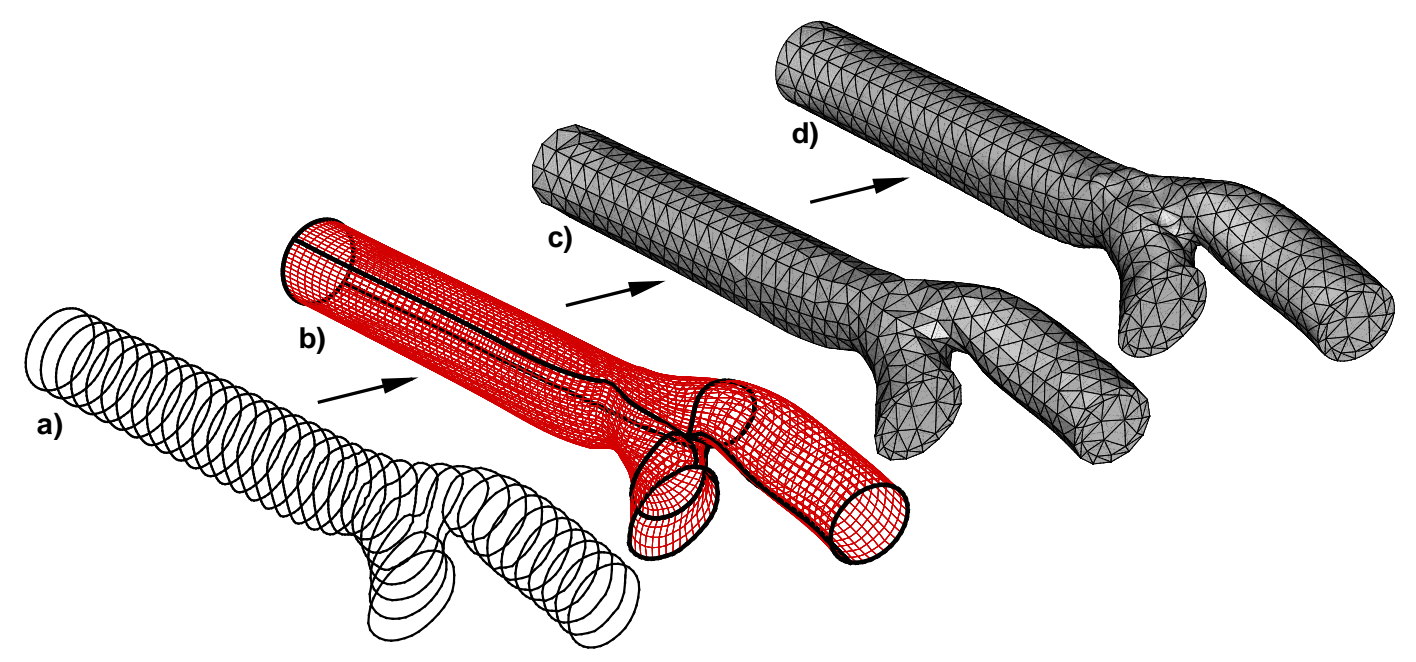

Figure 1. Construction of a high order computational mesh of an arterial bypass graft: a) smoothed slices of MRI data, b) contiguous surface representations, c) unstructured low order mesh and d) unstructured high order mesh.

Spectral/hp element methods combine the high accuracy and good phase properties of spectral methods with the geometric flexibility of the finite element method [3]. Traditionally these methods were developed in conforming structured sub-domains. The development of non-conforming [4] and unstructured approaches [5] have encouraged their use in more geometrically challenging problems [6]. These developments also naturally lend themselves to automated mesh generation to make them easier for a non-specialist to use. Defining robustness as the ability of a non-specialist user to apply the technique, then robust two-dimensional spectral $/ h p$ element solvers do exist. The same is not however true for three-dimensional problems.

Although the three-dimensional extension of these algorithms has already been addressed $[2,5,3]$, the amalgamation of such algorithms within three-dimensional mesh generation and CAD surface representation techniques has received little attention. The extension of current unstructured mesh generation technology to high-order algorithms is not trivial. Complications arise due to the conflicting requirements to generate coarse meshes whilst maintaining good elemental properties in regions of high curvature.

As an example of the types of configurations we wish to represent using high-order methods we consider the case shown in figure 1. It illustrates the construction of a high-order mesh representing a physiologically correct arterial bypass graft. The starting point for such a reconstruction is typically a set of slices of Magnetic Resonance Image (MRI) data. Each slice of MRI data provides a plane of information with pixels of different intensity corresponding to 
the response to a magnetic field. The values of the intensity are then thresholded and smoothed to produce the boundary of the domain of interest as shown in figure 1(a). From this data we construct a piecewise contiguous surface representation by means of tensor product spline surfaces [7] as shown in figure 1(b). This provides the necessary input information for the mesh generator to produce a coarse surface triangulation, as shown in figure $1(\mathrm{c})$, which is then transformed into a high-order surface mesh using the procedure described in section 1 as depicted in figure $1(\mathrm{~d})$. Having obtained a high order representation of the surface a volume mesh is then generated using standard linear element technology.

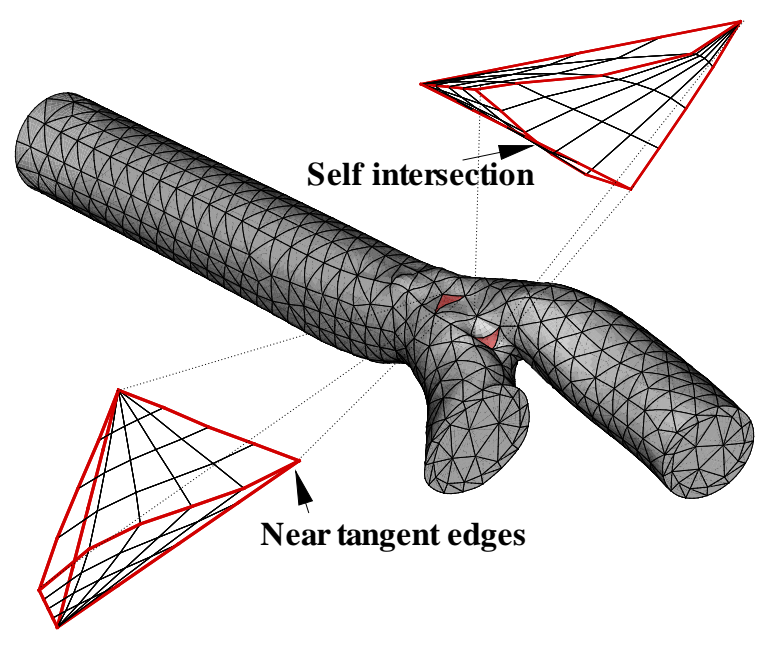

Figure 2. Singular element in high order tetrahedral mesh

Although figure $1(\mathrm{~d})$ shows a good surface representation of the desired computational domain, the final high-order discretisation is unacceptable since it contains elemental subdomains with singular local to global mappings. Two cases are illustrated in figure 2 . In the first case a singular mapping is generated as two edges become almost tangential to each other in the vicinity of a vertex. In the second case, the face of a tetrahedron intersects another face.

In this paper we will discuss three strategies to alleviate the problem of invalid high order meshes:

1. optimisation of the surface mesh generation,

2. hybrid meshing with prismatic elements near the domain boundaries, and

3. curvature driven surface mesh adaption.

We will illustrate these using the example of the biomedical computational domain shown in figure 1. In section 1 we review the development of unstructured mesh generation methods for high-order algorithms with particular attention to the spectral/hp element method. In section 2 we introduce surface optimisation for high-order surface mesh generation. In section 3 we demonstrate how the use of prismatic elements can reduce the appearance of distorted 
elements near the boundary and in section 4 we discuss the benefits of employing curvature driven surface meshing.

\section{Mesh generation of unstructured spectral/hp elements}

The method adopted to generate an unstructured mesh of spectral/ $h p$ elements, as originally presented in [8], proceeds in three steps. The first step is to generate a linear discretisation of triangular surface elements. The second step involves the transformation of that discretisation into high-order elements suitable for a spectral $/ h p$ computation. Finally the interior volume is constructed using standard low-order mesh technology although more advanced techniques may generally be necessary as discussed in Dey et al.[9].

The geometry of the computational domain is defined through a boundary representation (B-Rep) where the domain is viewed as the interior region to a boundary composed by a set of faces on surfaces intersecting along curves where the edges of the boundary lie. These curves and surfaces are described using standard techniques of computer aided design (CAD).

The generation of linear elements is achieved by using a modified advancing layers method in the near-wall regions and an advancing front technique in the rest of the domain as described in [10]. As mentioned previously, the first stage of the high-order meshing is to generate a coarse linear surface triangulisation and then to modify these sub-domains into a boundary conforming mesh of high-order elements by splitting the sides and faces in a bottom-up fashion which is consistent with a B-Rep of the computational domain.

The generation of high-order elements therefore starts with a discretisation of the sides of the triangular surface elements into $P$ points as required by a polynomial interpolation of degree $P-1$. There are two possible cases to consider. If the side belongs to a curve of the B-Rep, the intermediate $P-2$ points are placed along the length of the CAD curve where the edge lies. If the side is on a face of the B-Rep, then the intermediate points are positioned on the corresponding $\mathrm{CAD}$ surface. In both of these cases a point placement algorithm is required either within a parametric curve or surface. It is the generation of these points which we refer to as high-order mesh generation since this information is used to reconstruct a high order numerical representation of the computational domain.

At present we complete the volume generation of linear elements using the modified advancing layer combined with an advancing front technique. The higher order surface information and the ability to deform element internally could be utilised during the process although within the context of the current work this has not been necessary. For examples of high-order generation in structural mechanics problems requiring internal high-order generation see $[9,11]$.

An important part of the high-order mesh generation process is the construction of highorder edges and faces which conform to the CAD curves and surfaces of the B-Rep of the computational domain. To illustrate this process we consider the diagrammatic representation shown in figure 3. The starting point for the generation of a high-order representation of an elemental face belonging to a boundary is the definition of three vertices on a CAD surface. If an edge of the face lies on a CAD curve, its discretisation requires the placement of points along the CAD curve. Typically, a Gaussian quadrature type distribution is used. The shape of the other edges can be freely defined within the CAD surface. A computationally convenient approach is to use a linear interpolation between the parametric coordinates of the end points of the edge. 


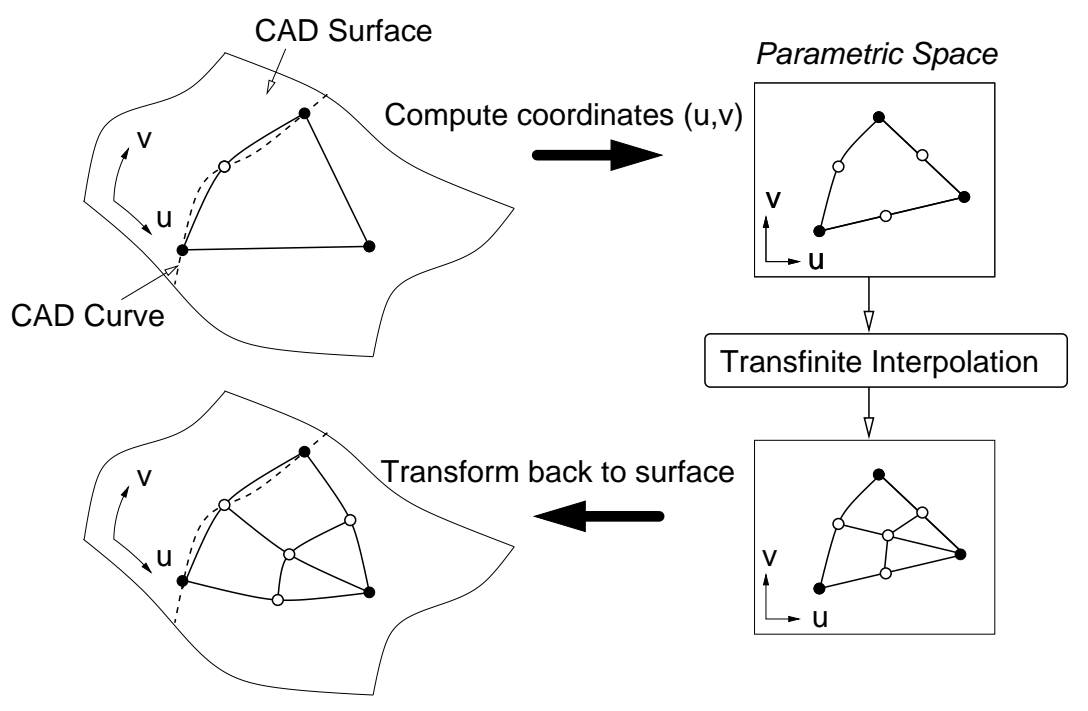

Figure 3. Mesh subdivision procedure

The points interior to the face can be determined in a similar fashion by using, for example, a transfinite interpolation within the parametric plane. Finally the three-dimensional coordinates of the boundary points of the elemental face are obtained by mapping the calculated parametric coordinates onto the CAD surface.

For CAD surfaces represented by isometric mappings (i.e. those that preserve lengths) the above procedure produces reasonably good quality elements. However, when the mapping is anisometric ${ }^{\dagger}$ this simple approach produces badly shaped elements and an alternative procedure is required to determine a suitable distribution of edge and face points. Such method is proposed in section 2 where optimal distribution of mesh points is calculated through minimisation of an appropriate cost function that represents the energy of the system.

\section{Optimising the surface approximation}

The algorithm presented in section 1 generated a high order surface representation by employing a transfinite interpolation between the discretised edges of triangles in the parametric space. This procedure does not take into account the mapping between the physical and parametric space and, in regions where the mapping is highly anisometric, such an approach can lead to very deformed mappings or, at worst, invalid elements.

To address the problem of obtaining an optimal distribution of points, let us consider a quadrature with $P$ integration points and associated normalised weights $z_{i} ; i=1, \ldots, P$ $\left(-1 \leq z_{i} \leq 1\right)$ in a one-dimensional interval $a \leq x \leq b$. It is known that the optimal positions

\footnotetext{
$\dagger$ Not isometric
} 
$x_{i} ; i=1, \ldots, P$ of the points are given by

$$
x_{i}=a\left(\frac{1-z_{i}}{2}\right)+b\left(\frac{1+z_{i}}{2}\right) \quad i=1, \ldots, P,
$$

since this leads to an isometric mapping and therefore a constant Jacobian. An optimal distribution of integration points for curves is easily obtained by interpreting the coordinate $x$ as the arc length of the curve. The implementation of this criterion using parametric CAD curves is relatively straightforward and amounts to finding the coordinates of a point in the curve that corresponds to the required length.

This procedure can be extended to elements with straight sides and faces by using a tensorproduct of optimal distributions along coordinate lines. However, a different strategy is required to achieve such an optimal distribution for curved edges and faces as we need to account for the distortion introduced by the presence of curvature.

A parametric surface is defined as a transformation between a two-dimensional parametric space $(u, v)$ and a three-dimensional space $\mathbf{r}(u, v)=[x(u, v), y(u, v), z(u, v)]$. Mesh generation is considerably simplified if performed in a parametric space. Therefore, as previously discussed, a computationally straightforward approach, illustrated in figure 3 , is to approximate the element edge by a straight line in the parametric plane and to distribute the points along that line according to equation (1). If the transformation $\mathbf{r}(u, v)$ is isometric, the mapped points on the surface will be appropriately distributed. A similar approach has been adopted in the work of Dey et al. [9]. However, if the transformation $\mathbf{r}(u, v)$ is anisometric, this approach can lead to highly deformed or invalid elemental regions.

\subsection{Optimal distribution of edge points}

A more general procedure can be obtained by reformulating the problem of finding the optimal distribution of points as that of minimising the potential energy of a set of springs linking adjacent points. If the stiffness of a spring joining nodes $i$ and $i+1$ is taken to be inversely proportional to the weight increment $z_{i+1}-z_{i}$, it is easily shown that the optimal distribution (1) is a minimum of the potential energy of such system of springs. For instance, in the onedimensional case the potential energy is given by

$$
\mathcal{J}\left(x_{2}, \ldots, x_{P-1}\right)=\sum_{i=1, P-1} \frac{\left(x_{i+1}-x_{i}\right)^{2}}{z_{i+1}-z_{i}} .
$$

This approach, unlike equation (1), is directly applicable to curved edges and faces on surfaces.

The implementation of this approach to edges on a parametric surface is slightly more involved. Since our parametric surface is defined in terms of the parametric coordinates $\mathbf{u}=(u, v)$, we recast the cost function as

$$
\mathcal{J}_{e}\left(\mathbf{u}_{2}, \ldots, \mathbf{u}_{P-1}\right)=\sum_{i=1}^{P-1} \frac{\left\|\mathbf{r}\left(u_{i+1}, v_{i+1}\right)-\mathbf{r}\left(u_{i}, v_{i}\right)\right\|^{2}}{z_{i+1}-z_{i}} .
$$

This is a two-dimensional minimisation procedure where the end points of the edge are fixed and the curve defining the edge is free to move in the parametric surface. The minimisation procedure is also non-linear since it involves the mapping $\mathbf{r}(u, v)$ between the physical coordinates and the parametric plane. 
The main advantage of using the minimisation approach rather than the transfinite interpolation is that the optimal solution will tend to a geodesic of the surface between the two end points. Further the distribution of points along the geodesic will also tend towards the optimal quadrature distribution. The cost function (2) can also be applied for discretising a curved edge defined as a function of a single parameter $u$. In this case the minimisation clearly does not alter the length of the curve and it will position the points to comply with the quadrature distribution (1).

\subsection{Optimal distribution of face points}

The previous method based upon an analogy with a spring network can also be applied to find the location of interior points within a curved elemental face. Assuming that a good distribution of points is known for a planar straight sided triangle, we can ask what is the set of constants $k$ in a network of springs that will produce the same distribution of points as its equilibrium position?

For this two-dimensional problem we define a discrete set of points within a standard region as illustrated in figure $4(\mathrm{a})$ for the case of a fifth order polynomial expansion (i.e. $P=6$ ). Introducing an interconnectivity between these nodal points as shown in figure 4(b) we develop a spring network such that minimising the potential energy of this system will position the physical points in a distribution similar to the original set of points in the standard region. This procedure aims at minimising the distortion of the mapping between the physical surface and the standard region. As we only know the optimal distribution of points for a planar triangle we use these points as a reference to calculate the spring constants. This is done as follows.

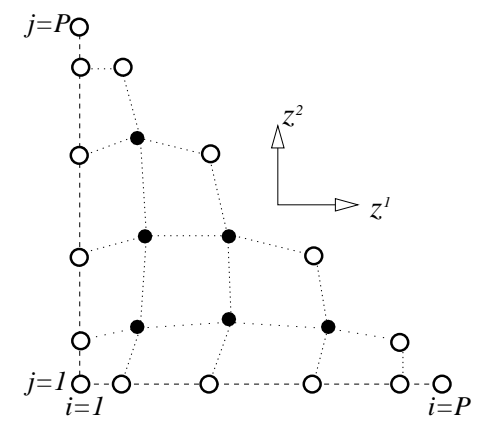

(a)

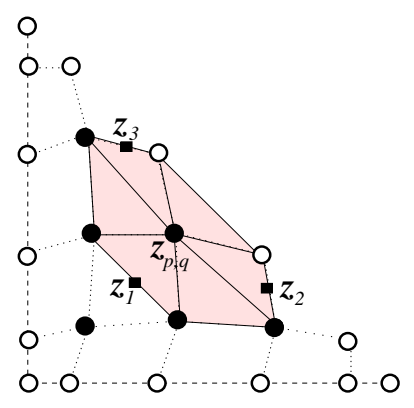

(b)

Figure 4. Ordering of barycentric points within (a) the standard right angle triangular element and (b) the equi-lateral triangle.

We consider a barycentric distribution of points in a standard region (for example $-1 \leq$ $\left.z^{1}, z^{2} ; z^{1}+z^{2} \leq 0\right)$ denoted by $\mathbf{z}_{i, j}=\left(z_{i, j}^{1}, z_{i, j}^{2}\right) ; 1 \leq i, j ; i+j \leq P+1$, as shown in figure 4 for $P=6$. To derive an expression for the spring constants we consider a general point $\mathbf{z}_{p, q}$ in the standard region also indicated in figure $4(\mathrm{~b})$. To maintain the symmetry of the point distribution we require that the desired point $\mathbf{z}_{p, q}$ is dependent upon the adjacent six points 
as indicated by the shaded region in figure 4(b). If initially we consider the adjacent points to be fixed, and making an analogy with a spring network, for point $\mathbf{z}_{p, q}$ to be at equilibrium we know that

$$
\begin{aligned}
\mathbf{F}_{p, q}= & k_{p-1, q}\left(\mathbf{z}_{p . q}-\mathbf{z}_{p-1, q}\right)+k_{p, q-1}\left(\mathbf{z}_{p, q}-\mathbf{z}_{p, q-1}\right)+k_{p+1, q-1}\left(\mathbf{z}_{p, q}-\mathbf{z}_{p+1, q-1}\right)+ \\
& k_{p+1, q}\left(\mathbf{z}_{p, q}-\mathbf{z}_{p+1, q}\right)+k_{p, q+1}\left(\mathbf{z}_{p, q}-\mathbf{z}_{p, q+1}\right)+k_{p-1, q+1}\left(\mathbf{z}_{p, q}-\mathbf{z}_{p-1, q+1}\right)=0
\end{aligned}
$$

where $\mathbf{z}_{i, j}$ follows an ordering convention as indicated in figure $4(\mathrm{a})$ and $k_{p-1, q}$ represents the spring constant between point $\mathbf{z}_{p . q}$ and $\mathbf{z}_{p-1, q}$. The other spring constants $k_{i, j}$ are defined analogously. To use this in a potential energy minimisation similar to the edge point positioning we need to evaluate the spring constants, $k_{i, j}$, which satisfy equation (3).

This evaluation is simplified by making the further assumption, which maintains the symmetry of the problem in the planar case, that

$$
k_{p-1, q}=k_{p, q-1}=\frac{k_{1}}{2}, \quad k_{p+1, q-1}=k_{p+1, q}=\frac{k_{2}}{2}, \quad k_{p, q+1}=k_{p-1, q+1}=\frac{k_{3}}{2} .
$$

This reduces the equilibrium problem to

$$
\mathbf{F}_{p, q}=k_{1}\left(\mathbf{z}_{p, q}-\mathbf{z}_{1}\right)+k_{2}\left(\mathbf{z}_{p, q}-\mathbf{z}_{2}\right)+k_{3}\left(\mathbf{z}_{p, q}-\mathbf{z}_{3}\right)=0
$$

where $\mathbf{z}_{1}=\frac{1}{2}\left(\mathbf{z}_{p-1, q}+\mathbf{z}_{p, q-1}\right), \mathbf{z}_{2}=\frac{1}{2}\left(\mathbf{z}_{p+1, q-1}+\mathbf{z}_{p+1, q}\right), \mathbf{z}_{3}=\frac{1}{2}\left(\mathbf{z}_{p, q+1}+\mathbf{z}_{p-1, q+1}\right)$ are the midpoints between the adjacent points, see figure 4(b). Finally, using the known position of $\mathbf{z}_{p, q}$ we evaluate the relative spring constants by taking the cross product of equation (4) with $\left(\mathbf{z}_{p . q}-\mathbf{z}_{2}\right)$ and $\left(\mathbf{z}_{p . q}-\mathbf{z}_{3}\right)$ to arrive at the conditions

$$
k_{1} \alpha=-k_{2} \beta \quad k_{1} \gamma=k_{3} \beta
$$

where

$$
\begin{aligned}
\alpha & =\left(\mathbf{z}_{p, q}-\mathbf{z}_{1}\right) \times\left(\mathbf{z}_{p, q}-\mathbf{z}_{3}\right), \\
\beta & =\left(\mathbf{z}_{p, q}-\mathbf{z}_{2}\right) \times\left(\mathbf{z}_{p, q}-\mathbf{z}_{3}\right), \\
\gamma & =\left(\mathbf{z}_{p, q}-\mathbf{z}_{1}\right) \times\left(\mathbf{z}_{p, q}-\mathbf{z}_{3}\right) .
\end{aligned}
$$

Condition (5) can clearly be satisfied by the choice:

$$
k_{1}=\beta, \quad k_{2}=-\alpha, \quad k_{3}=\gamma .
$$

We note that these conditions are also equivalent to the mechanical requirement that point $\mathbf{z}_{2}$ and $\mathbf{z}_{3}$ have zero applied moment which also implies zero moment about $\mathbf{z}_{1}$. The solution of 4 is equivalent to minimising the energy

$$
\mathcal{H}_{p, q}=\sum_{i=1}^{3} k_{i}\left\|\mathbf{z}_{p, q}-\mathbf{z}_{i}\right\|^{2}
$$

We now need to determine a set of $(P-3)(P-2) / 2$ interior points in the parametric space denoted by $\mathbf{u}_{i, j}=\left(u_{i, j}, v_{i, j}\right) ; 2 \leq i, j ; i+j \leq P$ such that the physical points $\mathbf{r}\left(u_{i, j}, v_{i, j}\right)$ have a similar distribution to the set of points $\mathbf{z}_{i, j}=\left(z_{i, j}^{1}, z_{i, j}^{2}\right) ; \leq i, j ; i+j \leq P$. By analogy to the planar case express in equation (7) we define the energy in terms of the physical space points $\mathbf{r}(u, v)$

$$
\mathcal{H}_{p, q}=\sum_{i=1}^{3} k_{i}\left\|\mathbf{r}\left(u_{p, q}, v_{p, q}\right)-\mathbf{r}_{i}\right\|^{2}
$$


where $k_{i}$ are defined by equation (6) and by analogy $\mathbf{r}_{i} i, 1, \ldots, 3$ is defined as:

$$
\begin{aligned}
& \mathbf{r}_{1}(u, v)=\frac{1}{2}\left[\mathbf{r}\left(u_{p-1, q}, v_{p-1, q}\right)+\mathbf{r}\left(u_{p, q-1}, v_{p, q-1}\right)\right] \\
& \mathbf{r}_{2}(u, v)=\frac{1}{2}\left[\mathbf{r}\left(u_{p+1, q-1}, v_{p+1, q-1}\right)+\mathbf{r}\left(u_{p+1, q}, v_{p+1, q}\right)\right] \\
& \mathbf{r}_{3}(u, v)=\frac{1}{2}\left[\mathbf{r}\left(u_{p, q+1}, v_{p, q+1}\right)+\mathbf{r}\left(u_{p-1, q+1}, v_{p-1, q+1}\right)\right]
\end{aligned}
$$

we have a general approach to determining the physical position $\mathbf{r}\left(u_{p, q}, v_{p, q}\right)$ in terms of its neighbouring points and the spring constants $k_{i}\left(z^{1}, z^{2}\right)$.

The optimal distribution for all the interior points, in terms of minimising the mapping Jacobian, is then obtained by minimising the sum of individual potential energies defined as

$$
\mathcal{J}_{f}\left(u_{2,2}, \ldots, v_{2,2}, \ldots\right)=\sum_{j=2}^{P-2} \sum_{i=2}^{P-j} \mathcal{H}_{i, j}
$$

The only assumptions we have made in terms of the desired location of the points is that they are barycentric and that the position of a single point is dependent on the six adjacent points both of which enforce rotational symmetry. Any distribution of points can be minimised in this fashion. Currently we have based the point location on the barycentric set proposed by Hesthaven [12] which are shown in figure 4 and are similar in distribution and properties to the points proposed by Chen \& Babuška [13].

We note that, although the distribution of points $[12,13]$ has near optimal properties in terms of approximation of interpolating polynomials, it is the placement of the points which define the mapping between the physical and standard triangular region and therefore the function to be approximated. It is therefore the choice of the points which minimises the distortion of the mapping from the physical plane to the standard local element which is important in maintaining the approximation integrity. However for cases where it is not possible to recover a constant Jacobian, for example on curved surfaces, the interpolation properties of these points are certainly also desirable. Finally we also make the observation that the points proposed by Hesthaven [12] have been derived by a similar minimisation process using an electrostatic potential. This approach could also be used directly in determining the high order mesh points within the surface.

\subsection{Surface Optimisation Results}

To illustrate the surface mesh generation methodology proposed in section 2, we consider the generation of a tetrahedral $p$-type mesh of fifth prder polynomial within a simple cubic computational domain $0 \leq x, y, z \leq 10$. The faces of the cube are located on tensor-product surfaces defined by an anisometric mapping shown in $5(\mathrm{a})$. The spacing varies linearly with a value of 0.1 at the boundary and 2.1 at the centre of the faces. The anisometry of the mapping is due to the fact that the unevenly spaced network of lines depicted in $5(\mathrm{a})$ is obtained as the image of a network of coordinate lines $u=$ const. and $v=$ const. in the parametric plane which are uniformly spaced with $\Delta u=\Delta v=1$. The curve definition of the edges representing the intersection between each of the faces was taken to be isometric.

A standard $h$-type unstructured mesh generation process was used to construct a coarse mesh with 66 elements. Although the $p$-type elements can easily be constructed on a planar 
(a)

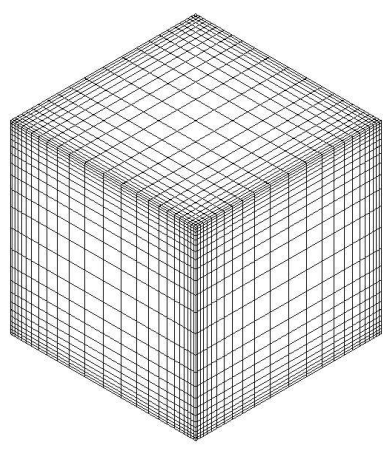

(b)

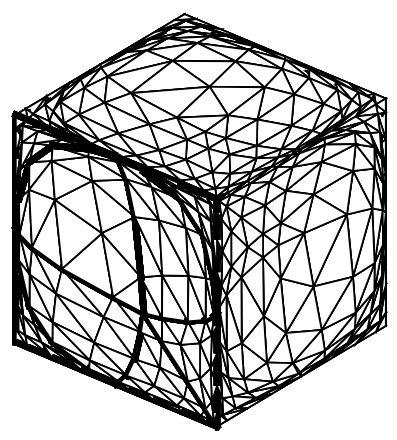

(c)
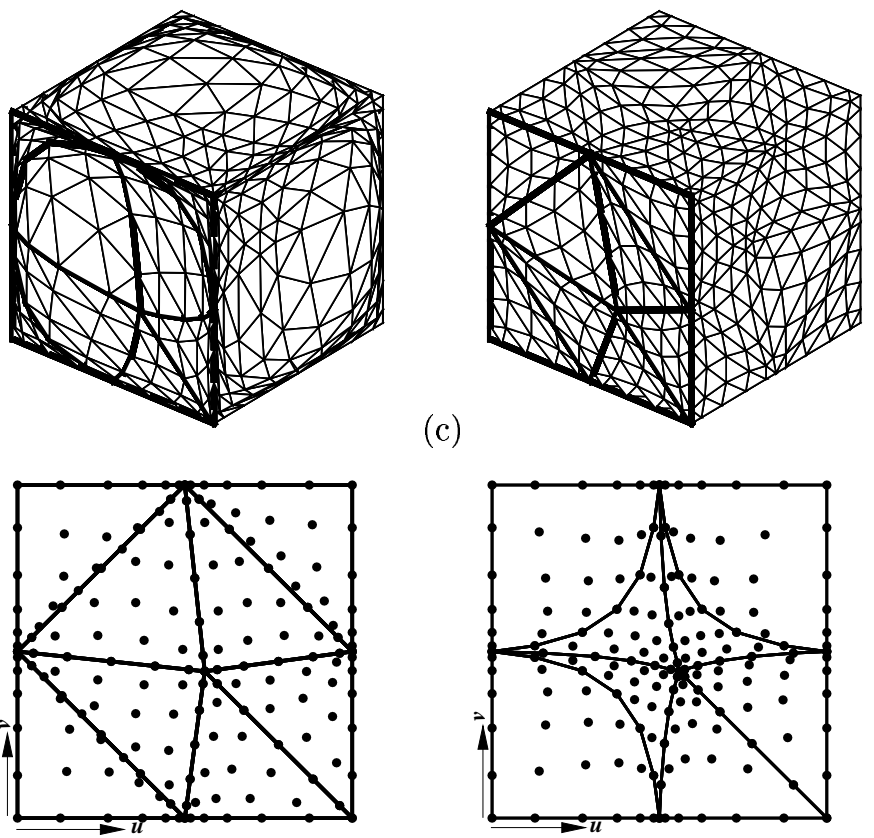

(e)

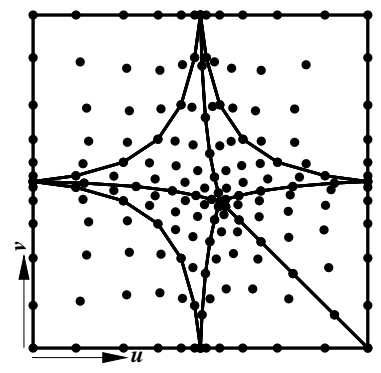

(f) (d)

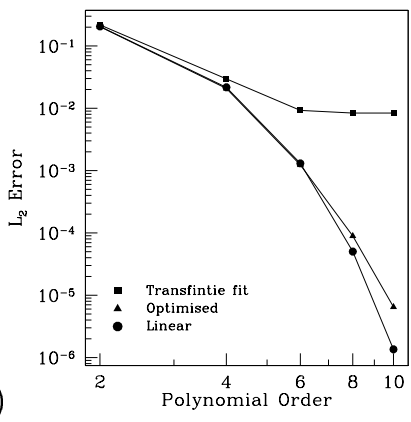

Figure 5. Influence of surface mapping on the $p$-type generation procedure. (a) Anisometric CAD description. (b) High-order mesh using a transfinite interpolation in the parametric space as shown in figure (e). (c) High-order mesh using an optimised point placement in the parametric space as shown in figure (f).(c) Comparison of errors in an elliptic problem using the different meshes.

surface by a linear interpolation between the vertices, we have chosen to reconstruct the surface elements using the parametric definition of the surface as required for a non-planar surface. Therefore we know that the optimal solution for this geometry is a linear distribution of points between the vertices according to the quadrature distribution given by equation (1).

Using a simple transfinite interpolation in the parametric space results in the highly distorted surface mesh shown in figure 5(b). In this figure we have connected all the interior edge and face points using a triangular mesh and highlighted the element boundaries on one face in dark lines. The distribution of points in the parametric plane for the highlighted surface are shown in figure $5(\mathrm{e})$. We note that the internal elemental regions have a regular distribution of surface points based on the point distribution of Hesthaven [12]. However the elements which touch the surface boundary have a more distorted distribution of interior points which is due to the non-quadrature spacing of the edge points on the surface boundary. This irregular distribution reflects the mapping between the isometrically defined edges and the anisometric definition of the faces.

However if we apply the optimisation procedure discussed in section 2, we obtain the surface mesh shown in figure 5(c) which produces a fit very close to a linear mapping between the vertices. A natural consequence of obtaining a good physical surface distribution is that the parametric distribution becomes very distorted as shown in figure 5(f). Finally, we compare 
the $L_{2}$ error to the solution $u(x, y, z)=\sin (0.2 \pi x) \sin (0.2 \pi y) \sin (0.2 \pi z)$ of a Poisson equation to assess the influence of mesh distortion on the solution accuracy. Figure $5(\mathrm{~d})$ compares the error of solutions obtained using computational meshes from a transfinite parametric fit (figure 5(b)) with those from an optimised parametric fit (figure 5(c)) and a standard linear fit between the physical vertices. The transfinite parametric fit leads to elements with sinbgular Jacobian and so it is not surprising that the error quickly saturates at $1 \times 10^{-2}$. The optimised interpolation, with a convergence tolerance in the parametric space of $\epsilon \propto 0.3 / P^{2}$, follows the error of the linear fit up to a polynomial order of $P=8$ where the error is of order $1 \times 10^{-4}$. For higher order expansions the rate of convergence decreases when compared to the standard linear discretisation. However increasing the convergence tolerance of the optimal iterative procedure reduces the saturation level.

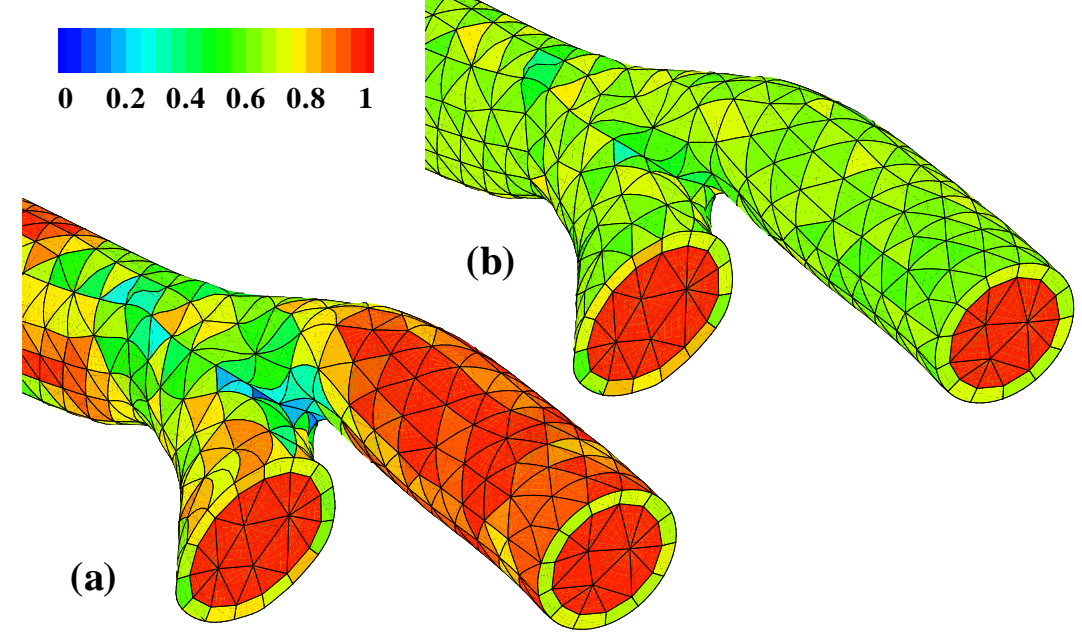

Figure 6. Surface Jacobian distribution with hybrid discretisation using (a) transfinite blend and (b) optimised point distribution.

As a final test we have applied the optimal surface discretisation to mesh the domain representing an arterial bypass graft shown in figure 6 . In this test we have adopted the measure proposed by Dey et al. [11] which characterises the variation of the elemental surface Jacobian by

$$
l=\frac{\min \left(J_{i}^{2 D}\right)}{\max \left(J_{i}^{2 D}\right)} \quad \forall i \in Q^{e} .
$$

where $Q^{e}$ represents the discrete set of quadrature points within the elemental surface and $J^{2 D}$ is the surface Jacobian. Figure 6 shows the values of $l$ for the hybrid discretisation of figure 2 before, figure 6(a), and after, figure 6(b), mesh optimisation. We are considering the two-dimensional surface Jacobian and all elemental regions are valid and non-singular. The triangular elements at the ends of the domain are all straight sided and therefore have a value of $l=1$. However, before surface optimisation, the minimum measure of the surface deformation 
was $l=0.1$ this is raised to a value of $l=0.32$ after applying the mesh optimisation. Further, there is a noticeable reduction in the level of surface deformation as indicated by the increased value of $l$ particularly in the vicinity of the junction.

\section{Hybrid Discretisation}

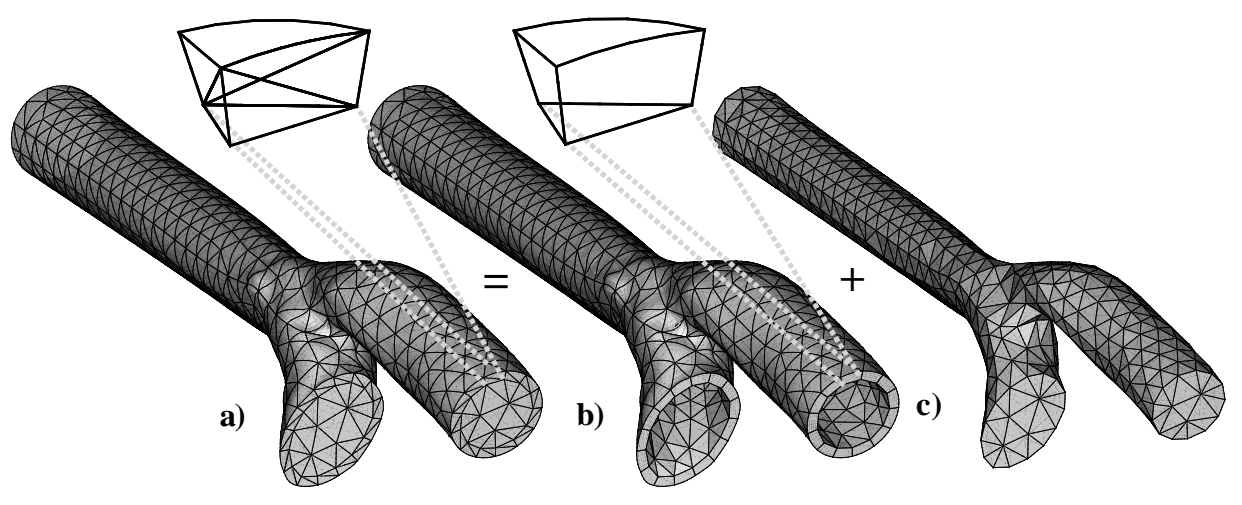

Figure 7. The boundary layer mesh adjacent to the solid boundary is typically constructed by extruding prismatic regions from the surface and then subdividing them into tetrahedra as shown in figure (a). Alternatively the domain can be discretised using a hybrid expansion of prismatic elements adjacent to the boundary, as in figure (b) which surrounds an inner core of tetrahedral elements shown in figure (c).

Inertially dominated viscous flows will invariably contain a boundary layer region near solid boundaries. Due to the rapid variation of the solution within this region it is common practice to use a "boundary layer" mesh [10]. An unstructured boundary layer mesh can be constructed by extruding the surface triangulation in the wall normal direction as shown in figure 7(a). This produces a series of prismatic regions that can be subdivided into three tetrahedral elements. Such an approach for low order meshes is described in [10] and the high-order meshing strategy discussed in section 1 can then be applied provided that the local surface is concave. However, in a locally convex region, the division of the prismatic regions into tetrahedral elements can lead to self intersecting elements as shown in figure 2. An obvious alternative is not to subdivide the prismatic region but apply a hybrid expansion $[14,15,16]$. The computational domain then consists of an outer shell of prismatic regions surrounding an inner core of straight sided tetrahedral elements as shown in figures $7(\mathrm{~b})$ and (c). Since the inner tetrahedral domain contains only straight sided elements, the sub-domains are guaranteed to be valid if a valid low-order mesh has been constructed. An alternative strategy to generate valid tetrahedral meshes would be to apply mesh modification involving internal face and edge swapping and/or 
deformation as proposed by Dey et al. [11].

For the computational domain depicted in figure 1, the introduction of prismatic regions, as shown in figure 8, reduces the number of sub-domains with singular mappings from 21 to 2. However, singular prismatic regions may still be generated if the deformation of the surface within an element is larger than the boundary layer thickness. This is illustrated in figure $8(\mathrm{~b})$. These elements can be corrected by internal face deformation. This can however be undesirable as internal face deformation can lead to a large number of elements around the face having nonuniform Jacobians. This could potentially reduce the accuracy of the local approximations. An alternative strategy is to apply curvature based surface discretisation. This will be discussed in section 4 .

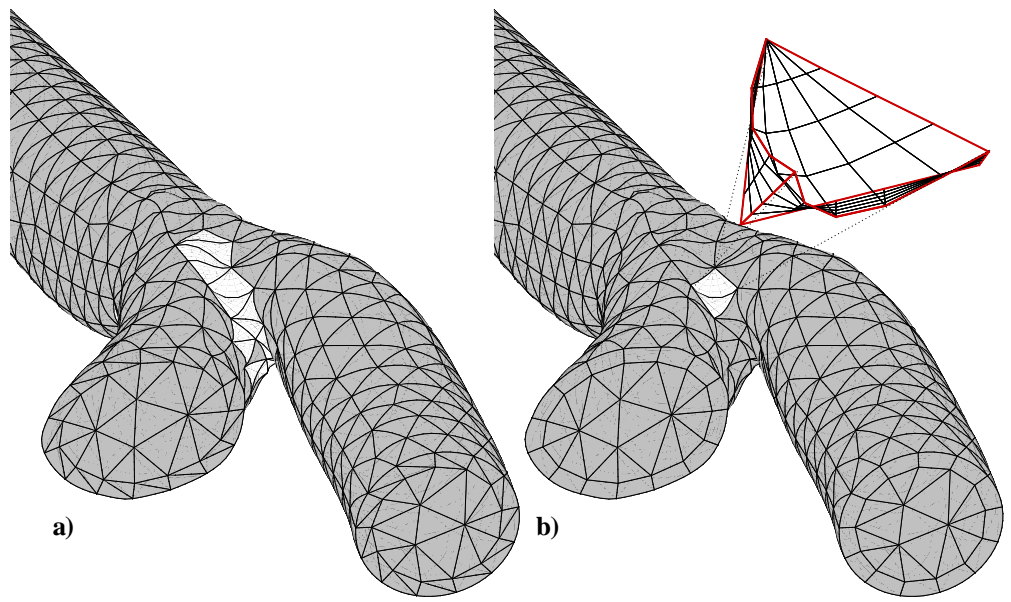

Figure 8. Invalid elemental regions (white) within (a) a tetrahedral mesh and (b) a hybrid discretisation. Also shown in figure (b) is an enlargement of an invalid prismatic element.

Finally, we note that the construction of the boundary layer mesh can be problematic in certain geometric configurations. For instance, along the line where two surfaces meet the surface normal might be multi-valued. In order to produce a continuous boundary layer description the normals within each plane are typically averaged. However, in the vicinity of a sharp external corner such as the trailing edge of an aerofoil, averaging of the normals can lead to highly distorted local prismatic regions. We should however stress that this problem is an issue for standard mesh generation using a boundary layer meshes. It is not specific to high-order meshing. 


\section{Curvature based refinement}

As discussed in section 3, hybrid discretisations using prismatic rather than tetrahedral elements in the boundary layer region can significantly alleviate the occurrence of invalid elements. However the standard low order boundary layer generators do not take into account the surface curvature and so it is still possible to generate invalid prismatic elements as shown in figure 2. Since high local curvature is a good indicator of where this problem is likely to occur an intuitive solution is to apply curvature based refinement to the surface discretisation.

Following the notation of figure $9(\mathrm{a})$, the curve is locally approximated by a circle of radius $R$, the radius of curvature. We assume that the mesh spacing can be represented by a chord of length $c$ in the circle. Denoting the maximum distance between the chord and the circle by $t$, we restrict its value, as suggested in [17], to $\frac{t}{R} \leq \epsilon$, where $\epsilon$ is a user defined tolerance. The mesh spacing, $\delta$, can now be obtained as a function of $R$ and $\epsilon$ as

$$
\delta \approx c \leq 2 R \sqrt{\epsilon(2-\epsilon)} .
$$

\subsection{Curves}

The radius of curvature of a curve $\mathbf{r}(u)$ is given, eg in [18], by the expression

$$
R^{2}=\frac{\left\|\mathbf{r}_{u}\right\|^{\frac{3}{2}}}{\left\|\mathbf{r}_{u} \times \mathbf{r}_{u u}\right\|}
$$

where the subscript indicates differentiation with respect to $u$. The mesh spacing can then be directly determined from equation (11) once $\epsilon$ is specified.

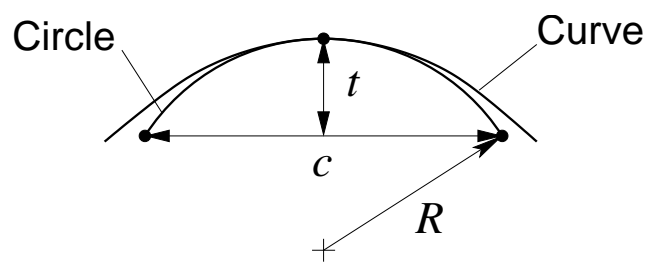

(a)

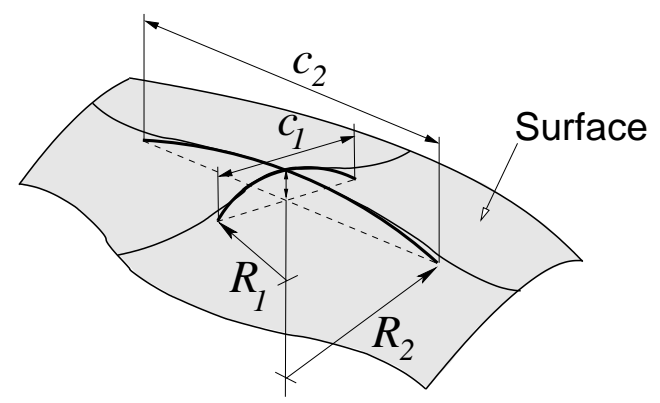

(b)

Figure 9. Notation for curvature calculation: (a) curves, (b) surfaces.

\subsection{Surfaces}

The curvature at a point on a surface $\mathbf{r}(u, v)$ can be characterised through the principal curvatures and directions of curvature. These are obtained as the eigenvalues and eigenvectors, respectively, of the matrix

$$
\mathbf{A}=-\mathbf{T}_{c} \mathbf{T}_{m}^{-1}
$$


where $\mathbf{T}_{m}$ and $\mathbf{T}_{c}$ denote the metric and curvature tensor of the surface and are given by

$$
\mathbf{T}_{m}=\left[\begin{array}{ll}
E & F \\
F & G
\end{array}\right]=\left[\begin{array}{ll}
\mathbf{r}_{u} \cdot \mathbf{r}_{u} & \mathbf{r}_{u} \cdot \mathbf{r}_{v} \\
\mathbf{r}_{v} \cdot \mathbf{r}_{u} & \mathbf{r}_{v} \cdot \mathbf{r}_{v}
\end{array}\right] ; \quad \mathbf{T}_{c}=\left[\begin{array}{cc}
e & f \\
f & g
\end{array}\right]=\left[\begin{array}{ll}
\mathbf{N} \cdot \mathbf{r}_{u u} & \mathbf{N} \cdot \mathbf{r}_{u v} \\
\mathbf{N} \cdot \mathbf{r}_{v u} & \mathbf{N} \cdot \mathbf{r}_{v v}
\end{array}\right]
$$

and $\mathbf{N}$ denotes the unit normal to the surface, i.e.

$$
\mathbf{N}=\frac{\mathbf{r}_{u} \times \mathbf{r}_{v}}{\left\|\mathbf{r}_{u} \times \mathbf{r}_{v}\right\|}
$$

The refinement criterion given by formula (11) is used for the two principal directions and the corresponding mesh spacings, $c_{1}$ and $c_{2}$ in figure $9(\mathrm{~b})$, are calculated from the values of the principal curvatures $k_{1,2}=1 / R_{1,2}$. Their values are given by

$$
k_{1,2}=H \pm \sqrt{H^{2}-K}: \quad\left\{\begin{array}{l}
K=\frac{\operatorname{det} \mathbf{T}_{c}}{\operatorname{det} \mathbf{T}_{m}} \\
H=\frac{e G-2 f F+g E}{\operatorname{det} \mathbf{T}_{m}} .
\end{array}\right.
$$

\subsection{Examples}

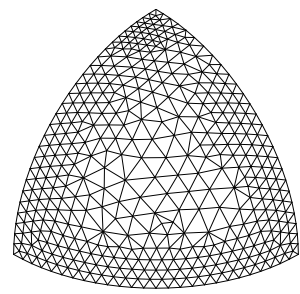

$\varepsilon=0.005$

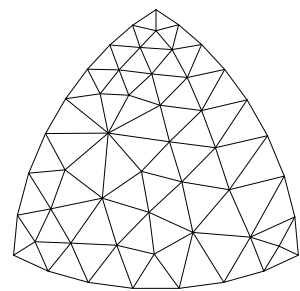

$\varepsilon=0.08$

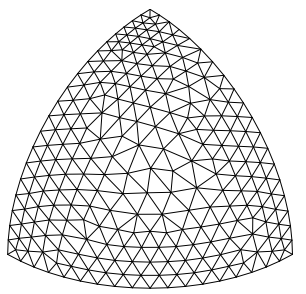

$\varepsilon=0.01$

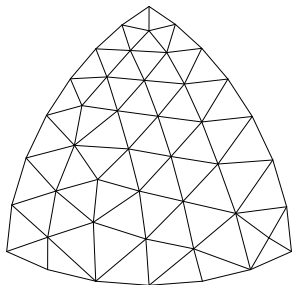

$\varepsilon=0.1$

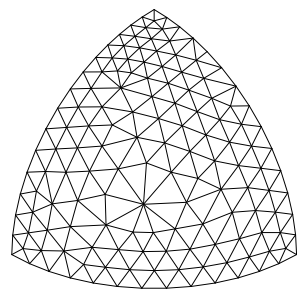

$\varepsilon=0.02$

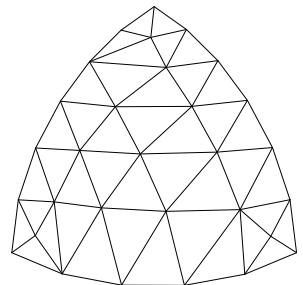

$\varepsilon=0.2$

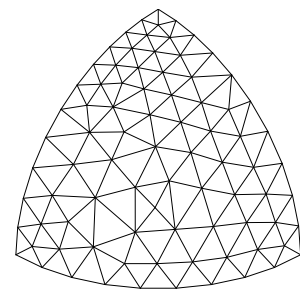

$\varepsilon=0.04$

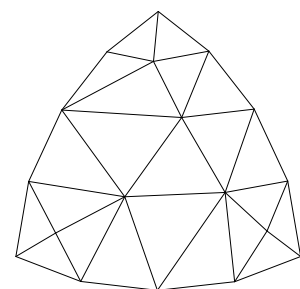

$\varepsilon=0.5$

Figure 10. Curvature driven surface refinement as a function of the tolerance $\epsilon$ (see equation (11)).

To get an indication of how the tolerance $\epsilon$ affects the surface resolution we can consider the case of a surface discretisation of a sphere as shown in figure 10. This figure shows the surface mesh resolution of a quadrant of a sphere as we increase the tolerance from $\epsilon=0.005$ to $\epsilon=0.5$. As a guide, a value of $\epsilon=0.01$ results in the division of a circle into 26 segments. 


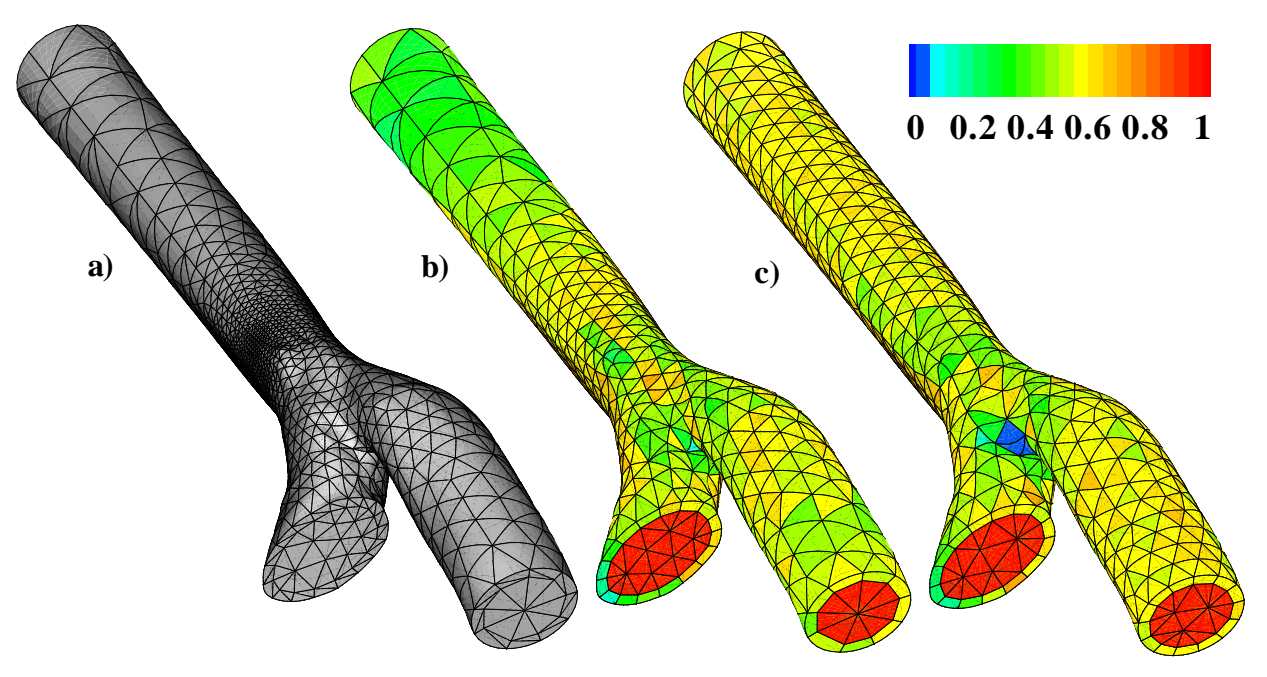

Figure 11. Curvature based surface refinement (a) no minimum element length (b) minimum element length imposed (c) no curvature refinement. Contour levels in (b) and (c) indicate variation in the local to global Jacobian.

Applying the curvature based surface refinement to the biomedical mesh we obtain the element distribution shown in figure 11(a). In this case a tolerance of $\epsilon=0.1$ was specified and subsequently the spacing was scaled by a factor of four. Accordinglly we note that the majority of the refinement is just above the junction where there is high curvature. Since the mesh generator does not permit a too rapid variation in element size, the effect of the refinement is to significantly increase the surface number of elements in this region. The final discretisation has 12,375 tetrahedral elements as compared to 5,930 tetrahedral elements in the original discretisation shown in figure 1. To stop excessive refinement a threshold has been implemented. This is illustrated in figure 11(b) where the modified procedure has been applied to the same case with a minimum element size of one as compared to the diameter of 15 (the spacing was also scaled by a factor of four). We have also applied the hybrid discretisation leading to a mesh of 2891 elements as compared with 3702 elements in the case shown in figure 11(c) and used previously. Figures 11(b) and (c) also show the surface plot of the variation of the local to global Jacobian using the measure

$$
l=\frac{\min \left(J_{i}^{3 D}\right)}{\max \left(J_{i}^{3 D}\right)} \quad \forall i \in Q^{e},
$$

where $Q^{e}$ represents the discrete set of quadrature points within the elemental and $J^{3 D}$ is the local to global Jacobian. As mentioned previously the mesh in figure 11(c) has a singular mapping and has a minimum discrete value of $l=2.7 \times 10^{-3}$ corresponding to a factor of 
370 variation in the elemental Jacobian. The mesh in figure 11(b) has a minimum value of $l=0.06$ corresponding to a factor of 17 variation in the elemental Jacobian. It is interesting to note that the curvature based refinement in figure 11(b) leads to a coarser mesh away from the junction where the variation in the Jacobian is larger. The reduced number of elemental regions and generation of all valid elements makes the curvature based refinement notably better than the uniformly refined case.

\section{Summary}

The development of robust unstructured high-order methods is currently limited by the inability to consistently generate valid computational meshes for complex geometries without user intervention. Traditional unstructured mesh generation techniques can provide a coarse linear discretisation of a complex geometry. However the subdivision of this mesh into a boundary conforming mesh of high-order elements requires careful handling due to the distortion that non-planar surfaces might introduce.

In this paper we have presented three complementary approaches for improving high-order meshes. The first is the adoption of hybrid discretisation using prismatic and tetrahedral elements. Secondly, an approach has been developed to generate optimal surface discretisations for high-order elements that accounts for surface curvature. This generation process minimises the element distortion due to the surface curvature based refinement by searching for the shortest geodesic line between two points whilst maintaining a Gaussian quadrature distribution. Finally the use of curvature has proven to be a very useful tool to enable valid high-order mesh generation.

Further, numerical computations of solutions to elliptic problems have demonstrated that the proposed surface optimisation can significantly increase solution accuracy with respect to methods that do not account for the anisometry of the CAD surface mapping. The importance of this is evident even for simple computational domains where our results show that the application of the optimisation procedure could produce a valid computational mesh where other approaches fail.

\section{ACKNOWLEDGEMENTS}

The authors would like to acknowledge support from the Bupa foundation and the Heartbeat charity. Computational support was also provided by the Imperial College Biomedical Visualisation System. We would also like to thank Dr Tim Warburton for his help in the development of the hybrid spectral $/ h p$ element code.

\section{REFERENCES}

1. S. K. Lele. Compact finite difference schemes with spectral-like resolution. J. Comp. Phys, 103:16-42, 1992.

2. B. Szabó and I. Babuška. Finite Element Analysis. Wiley, 1991.

3. G.E. Karniadakis and S.J. Sherwin. Spectral/hp Element Methods for CFD. Oxford University Press, 1999. 
4. C. Bernardi, Y. Maday, and A.T. Patera. A new nonconforming approach to domain decomposition: the mortar element method. In H. Brezis and J. L. Lions, editors, Nonlinear Partial Differential Equations and Their Applications. Pitman and Wiley, 1992.

5. S.J. Sherwin and G.E. Karniadakis. Tetrahedral $h p$ finite elements: Algorithms and flow simulations. $J$. Comp. Phys., 124:14-45, 1996.

6. S.J. Sherwin, O. Shah, D.J. Doorly, J. Peiro, Y. Papaharilaou, N. Watkins, C.G. Caro, and C.L. Dumoulin. The inflence of out-of-plane geometry on the flow within a distal end-to-side anastomosis. ASME J. Biomech., 122:1-10, 2000.

7. F. Schwerfirm. Final Year Project, Department of Aeronautics, Imperial College, 2000.

8. J. Peiró, O. Shah, D. J. Doorly, S. J. Sherwin, and C. G. Caro. Unstructured viscous mesh generation for haemodynamic flow simulation using high-order spectral elements. In 6th International Conference on Numerical Grid Generation in Computational Field Simulations, pages 457-466, 1998.

9. S. Dey, M.S. Shephard, and J. E. Flaherty. Geometry representation issues associated with p-version finite element computations. Comp. Meth. Appl. Mech. Engng., 150:39-55, 1997.

10. J. Peiró and A.I. Sayma. A 3-D unstructured multigrid Navier-Stokes solver. In K. W. Morton and M. J. Baines, editors, Numerical Methods for Fluid Dynamics V. Oxford University Press, 1995.

11. S. Dey, R.M. O'Bara, and M. Shephard. Curvilinear mesh generation in 3d. In Proceedings of the 8th International Meshing Roundtable, 1999. South Lake Tahoe, California.

12. J.S. Hesthaven. From electrostatics to almost optimal nodal sets for polynomial interpolation in a simplex. SIAM J. Numer. Anal., 35(2):65-676, 1998.

13. Qi Chen and Ivo Babuska. Approximate optimal points for polynomial interpolation of real functions in an interval and in a triangule. Comp. Meth. Appl. Mechs Engrg., 128:405-417, 1995.

14. S.J. Sherwin. Hierarchical $h p$ finite elements in hybrid domains. Finite Elements in Analysis and Design, 27:109-119, 1997

15. T.C.E. Warburton. Spectral/hp Methods on Polymorphic Multi-Domains: Algorithms and Applications. PhD thesis, Brown University, 1999.

16. S.J. Sherwin, T.C.E Warburton, and G.Em. Karniadakis. Spectral $/ h p$ methods for elliptic problems on hybrid grids. Contemporary Mathematics, 218:191-215, 1998.

17. P. Frey and P-L. George. Maillages. Editions Hermes, 1999.

18. M. P. Do Carmo. Differential Geometry of Curves and Surfaces. Prentice-Hall, 1976. 